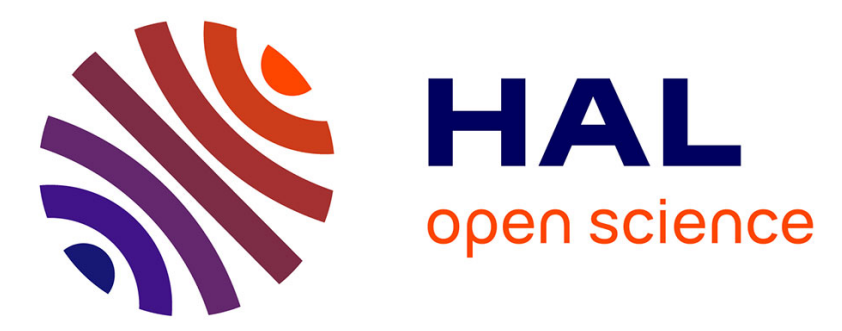

\title{
Du rôle des fêtes et de la joie comme moyens d'exciter la jeunesse à l'étude et de lui inspirer l'amour de l'école: le cas des Écoles du Dimanche françaises du XIXe siècle
} Anne Ruolt

\section{- To cite this version:}

Anne Ruolt. Du rôle des fêtes et de la joie comme moyens d'exciter la jeunesse à l'étude et de lui inspirer l'amour de l'école: le cas des Écoles du Dimanche françaises du XIXe siècle. Revue d'histoire et de philosophie religieuses, 2011, 91 (4), pp.525-548. 10.3406/rhpr.2011.1583 . halshs-00816164

\section{HAL Id: halshs-00816164 \\ https://shs.hal.science/halshs-00816164}

Submitted on 17 Jun 2016

HAL is a multi-disciplinary open access archive for the deposit and dissemination of scientific research documents, whether they are published or not. The documents may come from teaching and research institutions in France or abroad, or from public or private research centers.
L'archive ouverte pluridisciplinaire HAL, est destinée au dépôt et à la diffusion de documents scientifiques de niveau recherche, publiés ou non, émanant des établissements d'enseignement et de recherche français ou étrangers, des laboratoires publics ou privés.

\section{(ㄷ)(1) $\$$}

Distributed under a Creative Commons Attribution - NonCommerciall 4.0 International 


\title{
DU RÔLE DES FÊTES ET DE LA JOIE COMME MOYENS D'EXCITER LA JEUNESSE À L'ÉTUDE ET DE LUI INSPIRER L'AMOUR DE L'ÉCOLE
}

\section{Le cas des Écoles du Dimanche françaises du XIX ${ }^{e}$ siècle}

\author{
Anne Ruolt \\ Enseignante à l'Institut Biblique de Nogent \\ Post-doctorante, laboratoire CIVIIC, Université de Rouen
}

\begin{abstract}
Résumé: À partir de la littérature publiée par la Société des Écoles du Dimanche au XIX siècle en France, cet article considère les trois fêtes qui ponctuaient annuellement la vie des Écoles françaises. En milieu protestant orthodoxe, particulièrement rattaché au calvinisme ou au méthodisme, que montrent ces fêtes quant aux idées éducatives de ce dispositif?
\end{abstract}

Abstract: On the basis of the publications of the Society of Sunday Schools in $19^{\text {th }}$ century France, this article deals with the three feasts that yearly came back in the life of the French Sunday schools. We investigate in which way the celebration of these feasts in orthodox protestant circles attached to Calvinism or Methodism discloses the respective educational ideas of these schools.

Venant d'Angleterre, où le publiciste philanthrope Robert Raikes (1736-1811) l'avait lancé pour scolariser au moins a minima les enfants-ouvriers libres le dimanche, puis porté par le Réveil de Genève, le mouvement des Écoles du Dimanche (ÉdD) se déploie en France dès 1814 en terreau protestant orthodoxe nourri par le Réveil dissident britannique. L'objet de cet article se limite à la période 1852-1902, soit aux cinquante premières années d'action de la Société des Écoles du Dimanche (SÉD). Comparé aux traditions orthodoxe ou catholique, le culte protestant, dépouillé de rites, d'icônes, comme de clergé, frappe par son austérité apparente. Citant un pamphlet, Émile-Georges Léonard se fait le porte-parole d'une représentation classique du protestant :

Qu'est-ce qu'un protestant? Si le Français moyen ne le sait pas, la légende polémico-politique le sait, elle, et le lui dit. [...] Le protestant, c'est Calvin : vieux souvenirs de lycée ; Calvin le vieux prédicant aigre, ingrat, méchant, qui a brûlé Servet, empêché les Genevois de danser à 
leur gré $[\ldots]$. Et reste l'image du protestant : un dur, un coriace, peu maniable [...] d'un moralisme insupportable à «l'esprit gaulois » ${ }^{1}$.

N'est-il pas paradoxal, pour un dispositif éducatif marqué par le protestantisme, de parler des fêtes et de la joie comme moyen d'inciter à l'étude et à l'amour de l'école ${ }^{2}$ ? À moins que ces fêtes, à la suite du mouvement lui-même, soient plutôt une des marques du Réveil. En effet, en affublant les pasteurs réformés réveillés du sobriquet de mômiers ${ }^{3}$, les Suisses mettaient en exergue l'expression souvent très théâtrale et directe de ces prédicateurs. D'une autre façon, en Angleterre, les caricatures du pasteur baptiste et acteur du mouvement anglais des ÉdD, Ch. H. Spurgeon (1834-1892), témoignent en faveur d'un certain goût pour des formes d'expressions modérément figées et conventionnelles !

Selon une méthode herméneutique, et une approche a posterioriste, à partir des périodiques de la SÉD, le Magasin des Écoles $d u$ Dimanche (MagÉdD), suivi du Musée des Enfants (MusE), puis du Journal des Écoles du Dimanche (JÉdD), et de l'Essai sur les écoles $d u$ dimanche de Gauthey (1795-1864), cet article s'attache aux trois fêtes ponctuant le calendrier des écoles au XIX ${ }^{\mathrm{e}}$ siècle en France. La « fête générale » était régionale. C'était le point d'orgue qui, au printemps, au moment des Assemblées Générales (AG) des différentes œuvres de la grande famille protestante, commémorait l'anniversaire de l'œuvre et, par elle, de toutes les ouvres. L'histoire des fêtes de Paris de 1857 à 1863 a été consignée dans un ouvrage intitulé Les cirques, rédigé par un auteur-témoin désirant garder l'anonymat et signant : « un père de famille ${ }^{4}$. Fin décembre - début janvier, au niveau local, mais ouvert au public, la «fête de l'arbre » était l'occasion de récompenser publiquement les élèves les plus assidus.

\footnotetext{
${ }^{1}$ Léonard, 1953, p. 1-2.

${ }^{2}$ Ce titre est une paraphrase de De la vie dans les études ou Essai sur les moyens d'exciter la jeunesse au travail et de lui inspirer l'amour de ses devoirs, publié en 1860 par le pasteur-pédagogue L. F. F. Gauthey, ami de Pestalozzi à la fin de sa carrière à Yverdon, premier directeur de l'École Normale du Canton de Vaud puis de Courbevoie.

${ }^{3}$ Selon la feuille d'Avost citée par Ami Bost, cet usage date de 1818. "Lorsque la chaire fut interdite à Malan dans le territoire genevois, il se mit à prêcher de temps en temps à Fernex. Le 7 octobre 1818, l'article parut dans la Feuille d'Avos de Genève. Dimanche prochain, à Ferney-Voltaire, la troupe de mômiers, sous la direction du Sieur Régentin (M. Malan, ministre et régent au collège de Genève), continuera ses exercices de fantasmagorie, jongleries et tours de force simples. Le paillasse noir contribuera, par ses lazzis, à faire rire ses auditeurs. On trouvera des billets d'entrée près du bureau de la loterie. Cette ignoble plaisanterie suffit à mettre le mot à la mode dans le bas peuple » (Bost, 1854, t. 1, p. 100-101, 13). Dans le premier opuscule sur les ÉdD, écrit à Lausanne par le jeune Jean-Paul Cook, comme méthodiste, il n'hésite pas à s'approprier le terme " mômier » comme synonyme de "vrai chrétien. " Plus loin, Cook cite une situation où une jeune élève de l'ÉdD est insultée " par des garçons qui l'appelèrent une mômière " (Cook, 1847, p. 10 et 27).

${ }^{4}$ Père de famille, 1863.
} 
Au début de l'été, clôturant l'année, une " fête champêtre » rassemblait toute l'école pour vivre un événement récréatif.

Nous nous demanderons à présent ce que montre la scénarisation de ces fêtes quant aux idées éducatives de ces écoles.

\section{LA FÊTE GÉNÉRALE DES ÉCOLES DU DIMANCHE : « CULTE ANNIVERSAIRE » DANS DES LIEUX RÉSERVÉS AUX SPECTACLES !}

La « fête générale » est née timidement le jeudi 12 mars 1857 , à l'Oratoire du Louvre, qui était alors le plus grand temple de Paris (1500 places). C'est pour remercier le philanthrope américain Albert Woodruff ${ }^{5}$ (1807-1891), venu passer deux hivers à Paris pour encourager les ÉdD, que fut organisée cette grande rencontre festive pour les enfants des EdD, une façon de fêter aussi «l'anniversaire » de la SÉD à l'époque des $\mathrm{AG}^{6}$. Ces rencontres vont ensuite marquer de façon plus officielle la période des AG réunissant à Paris, à cette période de l'année, les membres des œuvres protestantes. Elles suscitèrent de nombreuses interrogations quant à la faisabilité du projet : comment trouver une date commune, un lieu approprié suffisamment vaste, des chants communs, mais aussi comment organiser des prises de parole intéressantes en les distribuant aux représentants des différentes dénominations ? Ces questions trouvèrent l'une après l'autre des réponses grâce à la volonté et aux efforts des acteurs parisiens de la SÉD. Le comité adressa une circulaire d'information aux responsables des trente-deux ÉdD de Paris et de sa banlieue, imprimant quatre nouveaux cantiques à deux voix.

Dès l'ouverture des portes du temple, à midi et demie, " un flot incessant d'enfants » convergea vers l'Oratoire. Le jour « $\mathrm{J} »$, 1858 élèves présents sont dénombrés, représentant 24 écoles et 6 dénominations ${ }^{7}$. Aux 500 instructeurs ou parents, Cook écrit qu'il faut encore ajouter environ les 2000 enfants venus spontanément

\footnotetext{
${ }^{5}$ Originaire de Sandisfield, le jeune A. Woodruff ne fit pas de longues études. À vingt ans, s'associant à son frère, il commença un commerce de poissons et de sel. Il allait à la Pilgrims-Church de Brooklyn, Église congrégationaliste, qui, dès 1840, était fréquentée par des intellectuels d'alors (Abraham Lincoln, Charles Dickens). Woodruff devint le premier responsable de son ÉdD, après en avoir été lui-même précédemment élève, puis moniteur. (Pavard, 2009, p. 359). Sans être théologien, en 1878, il fonda l'Association des ÉdD étrangères : Foreign Sunday School Association (FSSA) (Trumbull, 1888, p. 136, note 1; Voigt, 2007, p. 79). C'est au cours d'un agréable voyage en Europe, qu'il effectua en 1856 en famille, qu'il fut troublé, surtout à Paris, par la façon dont les Français " profanaient » le dimanche. Convaincu de l'impact de l'engagement des laïcs dans l'évangélisation, il pensa stratégique de promouvoir les EdD.

${ }^{6}$ Père de famille, 1863, p. 71-72; Cook, 1857.

${ }^{7}$ Église réformée : 9 écoles, 982 élèves; Église luthérienne : 5 écoles, 512 élèves ; Églises indépendantes : 6 écoles, 287 élèves ; Églises wesleyennes : 2 écoles, 35 élèves; Église anglicane : 1 école, 30 élèves ; Église baptiste : 1 école, 12 élèves (Cook, 1857, p. 105).
} 
sans conducteurs ni pasteurs mais encore : «quant au public, qui assiégeait en foule les portes du temple, il fut impossible de l'admettre, vu le manque de place ». Cet engouement montre l'intérêt suscité par ces rencontres bien au-delà des groupes d'enfants ${ }^{8}$. Dans les statistiques qu'il publie en 1864, Borel se rapporte aux dernières rencontres de la salle des fêtes du Trocadéro (actuellement Palais de Chaillot), où il estime que 4000 enfants étaient présents, sans compter leurs parents et autres adultes. Mais comparé aux rassemblements des ÉdD de New-York, dit-il, où 28000 enfants étaient réunis, les rencontres ne représentaient que peu de chose pour le philanthrope Albert Woodruff, qui créa l'occasion de la première de ces rencontres ${ }^{9}$.

Si le but de la première fête avait été de rassembler les enfants des ÉdD de la capitale pour remercier Wooddruff de sa visite en France, les fêtes suivantes commémorèrent l'anniversaire du mouvement et devinrent le moment festif, le "point d'orgue» des semaines où les différentes œuvres tenaient leurs AG à Paris ${ }^{10}$. Ces fêtes, drainant des foules, revêtaient aussi le caractère de "fêtevitrine » du protestantisme populaire et militant, sorti de la clandestinité, avec à présent la volonté d'influencer ouvertement la société. Relevons quant aux liens avec l'État que, dès 1858, celui-ci délégua la police pour assurer le maintien de l'ordre. Le 16 avril 1875, les musiciens du $68^{\mathrm{e}}$ de ligne, vinrent, sur l'invitation du gouverneur de Paris, prêter main forte à cette fête de l'enfance ${ }^{11}$.

Si l'iconographie souligne l'effet de masse, elle montre aussi la part prise par les adultes, et en particulier les pasteurs occupant l'estrade, dans ces rencontres à caractère à la fois solennel et familial. Considérant la minorité protestante, ce flot d'enfants accompagnés d'adultes venant écouter l'annonce de l'Évangile avait de quoi surprendre. À cela s'ajoutait l'étonnement devant les lieux retenus pour ces fêtes. Dans un pays catholique, de tradition sacramentaliste, l'annonce de l'Évangile dans des endroits habituellement réservés aux spectacles avait de quoi surprendre les Parisiens.

L'histoire garde la trace de semblables rassemblements de foule et de tels choix de lieux inédits pour prêcher l'évangile, mais en Angleterre : Wesley prêchait aux foules sur les places publiques ; le 19 octobre 1856, le jeune (il avait alors 22 ans) pasteur baptiste Charles Haddon Spurgeon (1834-1892) prêcha dans le « Surrey Music Hall », d'une capacité de 10000 places ; l'Armée du Salut prêchait

\footnotetext{
${ }^{8}$ Père de famille, 1863 , p. 71, 94.

${ }^{9}$ Borel, 1864, p. 42.

${ }^{10}$ Père de famille, 1863 , p. 71-72; Cook, 1857.

${ }^{11} A G S E ́ D, 1858$, p. $39 ; A G S E ́ D, 1875$.
} 
et chantait dans les bars dès 1855 en Angleterre, non sans susciter de l'incompréhension. En France, si de telles situations se sont encore présentées, elles évoquent le plus souvent des rencontres venues d'outre Atlantique comme celles qui eurent lieu de 1955 à 1986, au Vél d'Hiv et au Palais Omnisport de Bercy, où prêcha l'évangéliste américain Billy Graham. Mais qui se souvient que, dans la deuxième moitié du XIX ${ }^{\mathrm{e}}$ siècle, ce sont des pasteurs français qui rassemblèrent en grand nombre des enfants, pour des réunions organisées par la SÉD, qui préfigurent ce que Sébastien Fath surnomme Dieu XXL. La révolution des megachurches ${ }^{12}$ ?

En matière de pédagogie, il est à relever que les prédicateurs adoptaient un style d'enseignement plus direct que philosophique, même s'il était théologiquement moins raffiné. Sautter (1825-1912) rapporte la teneur de la tonique prédication du pasteur Auguste Decoppet (1836-1906), lors de la fête qui avait réuni le 5 juin 1890 , au Trocadéro, 3500 enfants. Adoptant pour rhétorique le procédé mnémotechnique, la chute du message, bien que vigoureuse, peut surprendre : «a, e, i, o, u » pour « apprendre, écouter, imiter, obéir et crier : hue ! en avant !» Ruben Saillens (1855-1942), qui représentait M. Mac-All (1821-1893), reprit ce même slogan, mais avec «U » comme Union, pour désigner ce qui caractérisait le plus cette fête selon lui. Puis, usant à son tour de l'analogie, en deux phrases, il exposait sa vision chrétienne mais non cléricale de la vie :

Enfants, je vois en chacun de vous un artiste qui a un tableau à faire et qui cherche son modèle. La toile, c'est votre vie ; les pinceaux, ce sont les facultés que Dieu vous a données ; les couleurs, le Saint-Esprit vous les fournira si vous les lui demandez; le modèle, c'est Jésus-Christ ${ }^{13}$.

Les accusations portées dès 1808 contre le pasteur whitefieldien Laurent Cadoret (1770-1861), fondateur de la première ÉdD française, le 7 août 1814 à Luneray, révélaient déjà un mode de prédication plus directe et proche de l'auditoire selon le modèle de la pratique des méthodistes, comme celle de son ami Du Pontavice (1770-1810) en Normandie ${ }^{14}$. C'était aussi le type classique de prédication de l'initiateur du méthodisme en France, Charles Cook (1787-1858). James Kendall fut étonné de cette autre façon de prêcher, plus directe et biblique que philosophique ou moralisatrice :

Ce fut en 1816, dit-il, que j'entendis pour la première fois un prédicateur wesleyen. [...] Accoutumé, comme membre de l'Église anglicane,

\footnotetext{
12 Fath, 2008.

${ }^{13}$ Sautter, $A G S E ́ D, 1891$, p. 6-7.

${ }^{14}$ Cadoret, 1808 , p. 1.
} 
à entendre lire des sermons du haut de la chaire, j'admirai la facilité avec laquelle il prêchait sans avoir de cahier sous les yeux ${ }^{15}$.

Alors que la tradition huguenote valorisait davantage le chant des Psaumes, en particulier ceux traduits par Clément Marot et Théodore de Bèze (1519-1605) dès le XVI ${ }^{\mathrm{e}}$ siècle, au sein de ces fêtes des ÉdD l'hymnologie est plus vive et contemporaine. Les pasteurs créent des chants adaptés aux circonstances : pas moins de quatre d'entre eux, à deux voix, sont imprimés pour la première fête du 12 mars 1857. Ajoutons à cela que le bestseller des Éditions de la SÉD en dit long : il s'agit du recueil de cantiques édité pour la première fois en 1857 en un volume de 72 pages, avec 83 cantiques $^{16}$. En 1938, une $41^{\mathrm{e}}$ édition devient un volume de 422 pages et 240 cantiques! Ce renouveau liturgique, qui se développe dans les ÉdD françaises par l'usage de cantiques d'un nouveau genre, était déjà à l'œuvre en Amérique du Nord dans les grandes réunions d'évangélisation, où prêchaient des revivalistes comme Moody (1837-1899), accompagné de son chantre Sankey (1840-1908).

Mais, comme le rappelle Alfred Kuen, cette évolution ne s'est pas faite sans heurt ni incompréhension. Pour lui, l'une des causes qui explique pourquoi le fossé se creuse de plus en plus entre la « musique d'Église » et la " musique profane » au $\mathrm{XIX}^{\mathrm{e}}$ siècle, c'est une certaine forme de ce que nous qualifierons de «biblolâtrie »: «En Angleterre, on considérait que chanter autre chose que des paroles bibliques était une erreur papiste ${ }^{17} \gg$. Un article, signé d'un auteur voulant garder l'anonymat (le sujet prêtant trop à polémique), présente clairement 1 'introduction de nouveaux cantiques auprès des enfants comme un moyen de réformer l'Église en commençant par les plus jeunes :

Un moyen facile d'ailleurs d'introduire de nouveaux airs dans le culte public est de les faire apprendre d'abord aux enfants, dans les écoles, et de les leur faire chanter ensuite dans les assemblées religieuses. Ce moyen est employé avec le succès le plus marqué dans l'Eglise de la confession d'Augsbourg à Paris, où, de cette manière, une assemblée nombreuse, qui n'a mềme sous les yeux que les paroles et non la musique des cantiques, s'habitue avec facilité et en très peu de temps à chanter des airs les plus mélodieux. [...] Outre cette Église, déjà les Églises françaises de la Confession d’Augsbourg, de Strasbourg, de Colmar et du Ban de la Roche, ont depuis longtemps adopté le chant des cantiques. Une nouvelle Église française de la Confession d'Augsbourg, établie depuis peu à Bocourt, département du Haut-Rhin, par le zèle de messieurs Japy, chefs d'une des plus belles manufactures d'horlogerie

\footnotetext{
${ }^{15}$ Cook, 1862, p. 22.

${ }^{16} \mathrm{D}$ 'autres recueils de cantiques avaient déjà été édités avant celui-ci, César Malan fut un auteur prolixe.

${ }^{17}$ Kuen, 2009 , p. $85,83$.
} 
de France, vient de l'adopter également. Le recueil dont elle se sert est celui de messieurs Ballif et Ebray ${ }^{18}$ de Bâle ${ }^{19}$.

Si la musique a été «l'une des principales voies d'accès à la culture pour les adultes comme pour les enfants », au XVIII ${ }^{\mathrm{e}}$ siècle, ainsi que le montre Loïc Chalmel ${ }^{20}$, sa fonction au sein des mouvements de Réveil est sociologiquement plus proche de l'usage en vigueur au sein des mouvements militants qui cherchent à faire pénétrer leurs valeurs auprès du peuple, pour changer les traditions établies par la base, sans utiliser la «musique savante ». Selon l'expression de William Booth (1829-1912), fondateur de 1'Armée du Salut, la musique peut être comparée, pour l'âme, à « ce que le vent est pour le navire, le poussant dans la direction sur laquelle on met le $\operatorname{cap}^{21} \gg$.

\section{LA FÊTE DE L'ARBRE : LE « NÉO-SYMBOLE HUGUENOT »}

Si la figure symbolique de l'arbre de Noël est d'origine germanique $^{22}$, c'est le mouvement des ÉdD qui a popularisé cette tradition en France $^{23}$. En 1859 déjà, Cramer évoquait la façon dont avaient lieu les fêtes autour d'arbres de Noël à Paris, mais aussi dans le Nord et à Livron, à «l'imitation des traditions allemandes ${ }^{24} »$. Jérôme Ripoull relève une première mention d'un sapin de Noël, installé en 1738 dans le château de Versailles par Marie Leszczynska, épouse lorraine de Louis $\mathrm{XV}^{25}$. En 1837, une autre mention est à relever, attestant l'origine rhénane de la tradition du sapin de Noël décoré, à l'occasion du mariage du duc d'Orléans avec la princesse protestante Hélène de Mecklembourg-Schwerin (1814-1858), princesse dont le soutien aux œuvres protestantes a été remarqué ${ }^{26}$. Dans une lettre du 8 juin $1845^{27}$, cette dernière mentionne une visite

\footnotetext{
${ }^{18}$ Cet article cite les noms d'autres recueils, celui du pasteur Gambs et celui du pasteur Henry de Berlin.

${ }^{19}$ Anonyme, 1818 , p. 344-345.

20 « Musique et pédagogie », Chalmel, 2004, p. 106-108.

${ }^{21}$ Boon, 1978, p. 115.

${ }^{22}$ Gauthey, 1858, p. 177 sq ; Cullmann, 1993.

${ }^{23}$ Une première mention figure déjà au livre de comptes de la Bibliothèque Humaniste de Sélestat, en date du 21 décembre 1521 (Ville-Sélestat, [2009]).

${ }^{24}$ Cramer, 1859 , p. 74-76.

${ }^{25}$ Ripoull, 2007, p. 29.

${ }^{26}$ Voir à ce propos, l'ouvrage à paraître de José Loncke, dont le manuscrit a obtenu en 2009, un prix «coup de cœur» dans le cadre du concours Jean Calvin lancé par les éditions La Cause.

${ }^{27}$ Mecklembourg-Schwerin, 1859, p. 205.
} 
d'encouragement faite à l'occasion d'une rencontre d'enfants des ÉdD organisée au cirque des Champs-Élysées ${ }^{28}$.

Gonin comparait volontiers le sapin à la croix huguenote : " comme les croix huguenotes sont venues du midi, les sapins de Noël également chers aux cœur protestants ont leur origine dans l'Est ${ }^{29}$ ». " Inconnues en France, il y a quarante ans », rapporte le rédacteur du $J E ́ d D$, en 1898 , « ces fêtes y sont devenues, au sein de nos Églises, une institution très populaire, également aimée des parents et des enfants ${ }^{30} »$. Le MagÉdD décrit ainsi une de ces fêtes mémorables, organisée à Paris, au Gymnase de M. Triat en 1857 :

Le jeudi 24 décembre 1857, à 7 heures et demie du soir, plusieurs écoles du dimanche de Paris se sont réunies en séance extraordinaire dans un magnifique local, le gymnase normal de M. Triat, avenue de Montaigne, $n^{\circ} 36$ (quartier Champs-Élysées), généreusement et gratuitement offert par le directeur pour la célébration préliminaire et enfantine de la grande fête du lendemain. Il s'agissait d'un Arbre de Noël, préparé à frais communs, pour les enfants de ces écoles du dimanche ${ }^{31}$.

Bien que faisant aujourd'hui plutôt partie du folklore de fin d'année, les arbres de Noël étaient à l'époque une innovation, attribuée à l'héritage protestant, comme les crèches de Noël sont rattachées à un héritage d'origine catholique ${ }^{32}$. Le rejet des statues et de leurs vénérations, mais aussi de la notion de "sainte famille» dans le protestantisme, fait de cet arbre qui «crée l'événement» le symbole d'une allégorie naturelle, illustrant le sens du message du salut clairement exprimé dans les Évangiles. Il n'y a cependant aucun fondement «biblique» à ce symbole, pas plus qu'à la «croix huguenote ». L'allégorie s'explique de la sorte pour Rouillard: l'arbre garni de pommes ${ }^{33}$ renvoie à «l'arbre du fruit défendu » de la Genèse, par lequel, en Éden, le mal entra dans le monde, et avec lui la rupture de l'Alliance avec le Créateur ${ }^{34}$. Mais cette lecture, à

\footnotetext{
${ }^{28}$ Le Cirque des Champs-Élysées ou cirque d'été fut érigé en 1841 dans le VIII ${ }^{\mathrm{e}}$ arrondissement de Paris, onze ans avant le Cirque Napoléon, ou cirque d'hiver (1852), construit dans le XI $\mathrm{e}^{\mathrm{e}}$ arrondissement. Les deux bâtiments étaient l'œuvre de l'architecte Jacques Hittorff. Le premier cirque avait une capacité d'accueil de 6000 places ; le second, de 4000.

${ }^{29}$ Gonin, 1963, p. 122-136.

${ }^{30}$ Anonyme, 1898.

31 Anonyme, 1858.

${ }^{32}$ C'est à François d'Assise que l'on attribue la paternité de la coutume. En 1223, pour illustrer le récit de la nativité, dans l'église de Grecchio, ce dernier avait créé la première " crèche vivante ". Au XVI ${ }^{\mathrm{e}}$ siècle, ce sont les Jésuites qui créèrent les premières crèches avec figurines. La tradition provençale est datée de 1803. Voir Ripert, 1956, p. 14.

${ }^{33}$ Le lien entre « le mal » et « la pomme » n'a cependant rien de fondé dans la Bible. Il tire son origine d'un jeu de mot en latin. La pomme et mal sont des homonymes dans cette langue : malus désigne à la fois le pommier et ce qui est mauvais, malum, la pomme et le mal. En langue française, comme en hébreu, les deux mots ne sont pas homonymes. L'association symbolique du « fruit défendu » avec la pomme est donc une erreur d'interprétation sans fondement linguistique cohérent. Voir Blocher, 1979, p. 121.

${ }^{34}$ Rouillard, 2003, p. 20.
} 
notre connaissance, n'est pas partagée par les premières ÉdD. Cellesci ne garnissaient l'arbre que de bougies ! Pour les ÉdD, l'arbre vert symbolisait plutôt le croyant, présenté par le psalmiste comme un « arbre toujours vert» $(P s$ 1). Les lumières qui l'ornaient rappelaient, quant à elles, l'incarnation du Christ, " lumière du monde » (Jn 8,12), ou « rayonnement de la gloire du Père » $(\mathrm{He} 1,3)$, venu révéler Dieu le Père et sauver l'homme du péché.

Dans le MagÉdD de 1861, c'est en lien avec un ouvrage du pasteur Bungener qu'est présentée une de ces fêtes de Noël, qui réservait à l'arbre de Noël une mise en scène toute particulière ${ }^{35}$. Celle-ci devait contribuer à éveiller progressivement à de bons sentiments et à endiguer le risque d'agitation. Nous en reproduisons quelques extraits :

L'obscurité si ardemment désirée arriva enfin; un chœur de moniteurs et de monitrices exécuta avec ensemble un hymne de Noël; à un signal donné une petite porte s'ouvrit et laissa voir une brillante clarté, puis tout à coup un arbre magnifiquement illuminé fit son entrée triomphale sur un petit char décoré de guirlandes de verdures et traîné par des petits garçons [...] ce beau sapin toujours vert [...] n'est-il pas l'image de la vie ? [...] N'est-il pas dit de l'homme qui prend son plaisir dans la loi de l'Eternel : «Qu'il sera comme un arbre duquel le feuillage ne flétrit point ». [...] Avez-vous observé ces enfants qui se pressaient tout à l'heure autour de nous pour que nous leur distribuions quelques-uns de ces présents qu'ils convoitaient déjà depuis un moment et qu'ils attendaient avec impatience? N'est-ce pas ainsi qu'ils se regroupent autour de nous chaque dimanche, pour que nous leur donnions un présent qu'ils devront garder toujours ? [...] Renouvelons chaque semaine cette belle fête de Noël. Notre arbre, c'est la Bible ${ }^{36}$.

Pourtant, le primat de la joie simple mais tonique qui se dégage de cette fête n'obère en rien ni son côté solennel ni son biblocentrisme, puisqu'au cœur du programme, comme pour les fêtes qui se déroulent alors au Cirque Napoléon et au Trocadéro, la teneur des discours qui alternent avec les chants consiste en des enseignements bibliques appliqués à la jeunesse. La synthèse du pasteur méthodiste Matthieu Lelièvre, rédacteur du $J E ́ d D$ le confirme, montrant aussi comment ce symbole, devenu huguenot, n'a pas été adopté sans résistance!

L'arbre de Noël est une innovation importée de l'étranger et qui a eu quelque peine à triompher des scrupules inspirés par la vieille austérité huguenote. Aujourd'hui, l'acclimatation est faite ; l'arbre de Noël a jeté de profondes racines dans les habitudes de nos Églises, et il en est bien peu où il ne s'allume quand revient le 25 décembre. Ce sont les enfants qui ont fait son succès et qui le maintiendront, en dépit des

\footnotetext{
${ }^{35}$ Voir aussi Gauthey, 1858, p. $179 s q .$.

${ }^{36}$ M., 1861, p. 46-48.
} 
gens austères. Il n'y a pas lieu de s'en affliger, à condition toutefois que l'on ne permette pas à cette institution de dévier de son but. Tout le programme de la fête peut tenir en deux mots. Qu'elle soit joyeuse et religieuse ! deux termes qui ne s'excluent pas, Dieu merci ! Noël est la fête de la joie, parce que c'est la fête du Sauveur. Que nos arbres de Noël apprennent donc à nos enfants, par une leçon de choses qui se gravera dans leur mémoire, que la religion de Jésus-Christ est la source de la joie la plus grande et la plus intarissable ${ }^{37}$ !

Ce type de fête était aussi organisé dans d'autres villes. Un témoignage rapporté par Ruben Saillens montre comment l'UCJG coopérait avec les ÉdD mais aussi comment les autorités civiles pouvaient aussi être coopératives. C'était à Lyon, en 1872 :

Pour célébrer Noël avec leurs élèves, jeunes gens et jeunes filles organisèrent une fête, pour laquelle ils demandèrent une des plus grandes salles de Lyon: le Palais Saint-Pierre. L'animateur, qui n'avait pas dix-huit ans, fut accueilli par le Maire, homme libéral, décidé à encourager toute initiative individuelle ou indépendante qui pouvait servir la morale et le relèvement du pays. D'ailleurs, le fils du Maire connaissait bien Ruben Saillens, travaillant lui aussi au Crédit Lyonnais. La demande fut donc accordée. Il ne restait plus qu'à trouver l'argent pour donner aux enfants des cadeaux de Noël. On en trouva grâce à la générosité des protestants de Lyon. [...] La fête fut un succès. Deux arbres de Noël furent dressés dans la grande salle. Les pasteurs de Lyon prêtèrent leur concours. Les enfants, guidés par les moniteurs et les monitrices, exécutèrent des chants bien réussis. Douze cents spectateurs remplissaient la salle, et de larges distributions de traités furent faites aux enfants et aux adultes ${ }^{38}$.

Mais cette audacieuse « icône-huguenote » avait ses détracteurs. En effet, dès 1898 , une monitrice interrogeait la rédaction du $J E ́ d D$ sur le bien-fondé de cette fête qui, selon elle, commençait à perdre son sens originel :

On ne peut guère assister à un arbre de Noël maintenant sans entendre des anecdotes tout à fait en dehors du sujet, et parfois triviales, dirais-je. Puis il y a la question des cadeaux, cause de grandes dépenses pour de pauvres petites écoles du Dimanche, sans compter la triste concurrence qui se fait à cet égard entre différentes églises d'une même localité. [...] Du reste par ce système de cadeaux, on arrive à blaser les enfants et souvent à les mécontenter, ainsi que les parents. Ne pourrait-on pas s'en tenir à une distribution de livres, brochures ou gravures, et à une distribution d'oranges qui aurait lieu après, dans la sacristie, afin de maintenir le respect du lieu de culte ${ }^{39}$ ?

Le constat fait l'année suivante par le pasteur Albert Valdez, d'Argenteuil, corrobore cet état de fait :

\footnotetext{
${ }^{37}$ Lelièvre, 1899 , p. 490.

${ }^{38}$ Wargenau-Saillens, 1847, p. 25.

${ }^{39}$ Une monitrice, dans Collectif, 1898, p. 469.
} 
La fête de l'Arbre tend, en effet, en bien des églises, à perdre de sa signification primitive. Elle est souvent l'occasion de dépenses exagérées ; les enfants, chaque année plus gâtés, deviennent de plus en plus exigeants. Quant aux fameuses historiettes, lorsqu'elles n'ont pas le défaut d'être quelque peu niaises, elles ne se rapportent pas toujours au sujet de Noël ${ }^{40}$.

La place qu'avait prise l'Arbre comme symbole au sein des ÉdD méritait une consultation démocratique plus large de la part des responsables de la SÉD. De l'avis général, la volonté de supprimer cette fête ne ressortait pas des courriers reçus. «Un seul déclare nettement qu'il faut bannir les arbres de Noël de nos temples où ils sont souvent une occasion de désordre et de scandale. » Un plaidoyer plus long remet simplement en cause la tenue de cette fête dans un lieu de culte ${ }^{41}$. Mais, s'appuyant sur les Réformateurs eux-mêmes, Louis Sautter (1825-1912), alors Président de la SÉD ${ }^{42}$, insistait sur la nécessaire " désacralisation » du lieu de culte protestant. Lorsque ces fêtes se déroulaient dans la salle de culte, n'avaient-elles pas comme avantage de faire pénétrer dans l'Église des personnes qui ne s'y seraient pas rendues dans d'autres circonstances?

Quant aux débordements qui pouvaient parfois avoir lieu au moment de l'illumination de l'arbre, c'est sur l'adage du dentiste, selon lequel il ne faut pas arracher mais plutôt guérir, que se fonde Sautter. C'est plutôt par une scénarisation de l'allumage qui prête au respect et à la solennité qu'il faut contrecarrer le capharnaüm, là où le laisser aller y a conduit. Ainsi, revenant à la métaphore familiale, l'ardent promoteur des ÉdD populaires prône l'effort éducatif plutôt que la suppression de la fête. Non sans pointe polémique, le Président ajoute que pour lui l'Église est non seulement une famille mais une famille joyeuse où les enfants expriment leur joie de vivre ! Le style direct du propos de Sautter mérite d'être cité :

Nous n'envoyons pas nos enfants dîner à la cuisine parce qu'ils ne se comportent pas à table aussi bien que nous le voudrions. Nous nous efforçons de leur donner de bonnes manières, et, avec de la fermeté et de la persévérance, nous réussissons toujours. Pasteurs et Directeurs d'Écoles du Dimanche, agissons de même avec nos élèves. Ne nous laissons pas démonter par leur indiscipline. Soyons patients et énergiques, et, par la grâce de Dieu, nous en viendrons à bout. Les petits Parisiens ne le cèdent en rien pour la turbulence et l'esprit éveillé aux petits Méridionaux. Ils nous ont fait bien des misères au début des Écoles populaires. Aujourd'hui, les fêtes d'Arbre de Noël de ces écoles ne sont pas moins calmes, moins bien ordonnées que celles de nos Églises

\footnotetext{
${ }^{40}$ Valdez, 1899, p. 491.

${ }^{41}$ Sautter, 1899.

${ }^{42}$ Louis Sautter n'était pas pasteur mais ingénieur diplômé de l'École Centrale de Paris. Il avait fondé une entreprise de phares. Collaborateur puis directeur de la Mission évangélique Mac-All, il fut nommé troisième président de la SÉD à la mort d'H. Paumier.
} 
aristocratiques de Paris, tout en étant, en général, plus joyeuses, parce qu'elles s'adressent à un public d'enfants moins blasés [...]. J'aime à voir les enfants venir, dès la première jeunesse, dans le sanctuaire où plus tard, ils accompagneront leurs parents, y faire provision de souvenirs qui le leur rendront cher pendant toute la vie. Si leur attitude n'est pas aussi grave, aussi réservée que celle des grandes personnes, si des explications leur sont données dans un langage à leur portée, si parfois une anecdote, un mot plaisant provoque dans le jeune auditoire un sourire, voire même, ô scandale ! un éclat de rire, quel mal y voyez-vous ? Aimeriez-vous mieux qu'il s'endormitt ${ }^{43}$ ?

Dans L'école dans la littérature, Claude Pujade-Renaud décrit le « rituel » bruyant et potache qui accompagnait dans les écoles la distribution des prix en fin d'année sur fond de «Les cahiers au feu et les maîtres au milieu ${ }^{44}$. Rien de tel dans les ÉdD d'après leurs propres sources. Les prix étaient distribués, à mi-parcours, à la fin du mois de décembre ou au début du mois de janvier et non en signe de couronnement de l'année. Ils ont pour but de stimuler le progrès de tous et ne sont pas une fin en soi. Comme pour les bons points, la crainte de leur assimilation à un salaire est prégnante au sein de la SÉD. Pour contrecarrer cette erreur, dans son Essai sur les écoles du dimanche, Gauthey pose le principe du devoir et de la dette. Comme on ne récompense pas qui « fait son devoir» en payant sa dette, il n'y a pas lieu de récompenser l'enfant qui fait «son devoir ». Cependant, reconnaissant que pour un jeune enfant, rester attentif à l'ÉdD est synonyme d'effort et de travail, la récompense a sa place, mais comme « un simple témoignage d'approbation, donné au nom du Seigneur et servant à déclarer que l'élève a été attentif, laborieux et exact ${ }^{45} \gg$.

Les trois critères d'évaluation privilégiés sont ainsi désignés : l'assiduité, la qualité du travail et la conduite, et non la performance intellectuelle de l'élève. Gauthey s'en explique en ce que, pour lui, le talent est un don de Dieu. Il appelle à la reconnaissance et à la responsabilité et non à une récompense. Aussi, l'évaluation ne se réduit pas non plus pour lui à une addition de chiffres ${ }^{46}$. L'enfant ne

${ }^{43}$ Sautter, 1899, p. 52 et 50.

${ }_{44}$ Pujade-Renaud, 2006, p. 37.

${ }^{45}$ Gauthey, 1858, p. 168.

${ }^{46}$ Gauthey, 1860, p. 37, note 1, 40. Vincent Troger situe au XVII ${ }^{\mathrm{e}}$ siècle, sur l'initiative des Jésuites, l'adoption des notes par les lycées (au départ de 1 à 6) ainsi que celle du classement des élèves. «Il s'agissait alors pour eux de former les élites intellectuelles chargées de lutter contre le protestantisme. Leur préoccupation était donc la distinction des meilleurs et non l'instruction de tous. En créant les lycées sur le modèle des collèges jésuites pour former les élites de l'État, Napoléon $\mathrm{I}^{\mathrm{er}}$ a maintenu cette culture de classement et de sélection. À l'école primaire, c'est la III ${ }^{\mathrm{e}}$ République qui, en voulant concurrencer les rituels catholiques, a donné un caractère très solennel aux classements : compositions mensuelles, tableaux d'honneur et d'excellence, remise des prix de fin d'année. » (Troger, 2008). 
devait pas pouvoir dire : " on va me récompenser d'après les bonnes notes que j'ai obtenues ${ }^{47} \gg$. La gratuité d'une récompense en est la notion cardinale ${ }^{48}$. L'on sent aussi, dans les propos du directeur de l'École Normale de Courbevoie, une certaine gêne devant la mise en scène des distributions de prix, qui flatte trop souvent l'orgueil des uns et en humilie d'autres. Il préconise davantage les paroles " paternelles » qui touchent l'affectif et renforcent les relations de confiance entre l'enfant et ses éducateurs, envisageant cette rencontre comme « une fête de famille ${ }^{49}$ ».

Des paroles affectueuses et familières, appropriées aux besoins de l'enfance et de la jeunesse, seraient prononcées par celui qui présiderait à la cérémonie ; on n'appellerait pas les élèves du nom de héros et de conquérants, comme cela est parfois arrivé ; on les mettrait à leur place ; on leur dirait que l'ornement de l'humilité est le plus beau de tous ; on les exhorterait à s'aimer, à vivre en bons camarades, et à profiter du jour de la fête présente, pour inaugurer un bon avenir. Les distributions, ainsi modifiées, n'offriraient plus les mêmes inconvénients ${ }^{50}$.

La récompense est alors synonyme d'approbation, ce que nous rapprochons du concept de "bénédiction» dans le sens grec de " dire du bien », eulogeô (et non dans un sens sacramentel). Ces paroles dites à propos sont telles «des pommes d'or dans des paniers d'argent », dit Gauthey ${ }^{51}$ en citant le livre des Proverbes $(25,11)$. Cette théorie des récompenses, ainsi définie, fait de l'homme non un héros autonome récompensé pour ses performances au détriment des autres moins " chanceux », mais plutôt le héraut $d u$ Créateur dont il est imago dei. Le développement progressif de son potentiel comme le caractère « vivant » et « créatif» de ses initiatives, qui ne se bornent pas à la « reproduction » mécanique des choses, en rendent témoignage. Si le potentiel peut croître, ce n'est qu'en vertu de la grâce dont tout homme est l'objet, la Providence l'ayant ainsi doté et placé dans un cadre où ses « forces » pourront se déployer en vue d'une croissance vigoureuse, qui dure pour la joie de tous.

Nous n'avons trouvé que chez Wilfred Monod, au tout début du $\mathrm{XX}^{\mathrm{e}}$ siècle, la mention d'exclusion des enfants de «mauvaise volonté » de cette fête comme mesure disciplinaire. Mais il précise : là où ces fêtes sont devenues des « institutions ». Faut-il entendre par là lorsqu'elles avaient perdu leur sens familial primitif ?

Dans presque toutes nos paroisses on allume, annuellement, un arbre de Noël ; parfois, le sapin est remplacé par une séance de projections

\footnotetext{
${ }^{47}$ Gauthey, 1858, p. 176.

${ }^{48}$ Gauthey, 1858, p. 166, 168.

${ }^{49}$ Gauthey, 1858, p. 180.

${ }^{50}$ Gauthey, 1860, p. 42-43.

${ }^{51}$ Gauthey, 1858, p. 180.
} 
lumineuses; en été, on organise, dans plusieurs de nos églises, une excursion à la campagne ou un goûter rustique. Là où ces diverses fêtes sont passées à l'état d'institutions, il est évident qu'on peut en écarter tel enfant dont la mauvaise volonté serait manifeste ${ }^{52}$.

Une autre mesure disciplinaire préconisée par Monod, selon le même principe de la privation, était la suspension du droit d'emprunter des livres à la bibliothèque " pour un laps de temps déterminé ». Mais priver sans autre l'élève indiscipliné de relation ou de plaisir de lire, est-ce lui faire plus aimer l'école ? Gauthey, en insistant plus sur l'effet positif produit par la joie et le plaisir, témoigne d'une approche éducative s'appuyant davantage sur l'affectif que ne le faisait W. Monod, plus soucieux du niveau d'instruction et plus influencé par les idées de l'école républicaine. Cette observation confirme le jugement de Jean-François Zorn :

On sent chez Monod l'influence de l'école publique et l'admiration qu'il portait aux instituteurs. C'est le modèle qu'il voulait voir appliquer aux moniteurs ${ }^{53}$.

\section{LA FÊTE CHAMPÊTRE DE FIN D’ ANNÉE, POUR RÉCRÉER ET STIMULER PAR LA GRÂCE}

Les « fêtes champêtres ", ouvertes à tous, avaient pour but de stimuler le zèle des enfants et de leur faire aimer les écoles en leur offrant un cadre détendu, hors de l'école, pour vivre autre chose. Récompenses récréatives après le travail, ces sorties clôturaient l'année. Comme un élément de la nature sert de symbole à la fête célébrée en hiver, en été les ÉdD vont au vert!

La première règle que fixe Gauthey pour les "récréations ", c'est d'avoir « quelque chose de naturel », conformément à la notion de « méthode naturelle » qui est la sienne ${ }^{54}$. La récréation doit aussi engager l'activité de l'enfant et non favoriser la passivité ${ }^{55}$. La dimension sociale, les valeurs de morale chrétienne, l'activité constituent les trois axes de la récréation, après le travail!

Cette récréation festive est synonyme d'égale récompense pour tous ! Le goûter et les jeux remplacent l'offre de littérature dans le déroulement de l'activité. En matière de jeux, le directeur de l'École Normale redoutait les enfants « trop sages » :

L'élève qui n'aime pas jouer avec ses camarades et qui préfère rester dans son coin, silencieux ou morose, n'est pas en général celui duquel

\footnotetext{
${ }_{53}^{52}$ Monod, 1904, p. 10.

${ }_{53}^{53}$ Zorn, $1996 / 3$, p. 385.

${ }^{54}$ Gauthey, 1854 , p. 399.

${ }^{55}$ Gauthey, 1861, p. 23, 33.
} 
on peut attendre les meilleures choses dans l'avenir. Cette eau qui dort annonce ordinairement des dispositions fâcheuses, si ce n'est des vices ${ }^{56}$.

Ainsi, les sorties sont 1'occasion de réjouissances détendues qui ont « le grand avantage de ne causer ni jalousie, ni récriminations ; tous sont contents, tous ont été heureux ensemble ${ }^{57} \gg$. Tous en bénéficient de la même façon, contrairement aux fêtes de fin de premier trimestre, qui récompensent les élèves selon des mérites. Si elles peuvent servir de moyen de discipline, c'est par le moyen de la privation pour l'élève indocile. Mais ce n'est pas ce que suggère Gauthey, qui y voit davantage un signe de miséricorde à l'endroit des élèves dissipés, faisant ressortir la « gratuité » de la récompense qui procède de la bonté et de la grâce et non du « salaire » ${ }^{58}$.

Offertes à tous, elles [les fêtes champêtres] ne mettent pas en jeu l'amour propre. Elles n'excluent point les éloges qui se donnent aux uns et les reproches qui sont adressés aux autres, lorsque le travail et la conduite de l'année sont l'objet d'un examen sérieux. Les élèves ne peuvent pas s'imaginer qu'on les met tous au même niveau et qu'ils sont tous compris, sans distinctions, dans une même approbation générale. Non, chacun sait que, s'il y a une fête, c'est aux bons sujets qu'on la doit et que, si les sujets médiocres y participent, c'est qu'on veut bien ne pas les exclure et troubler par des ombres la sérénité du beau jour qui va se lever. La conscience parle donc aux paresseux et aux turbulents. Ils se rappellent les torts dont ils se sont rendus coupables et, au besoin, l'œil de leurs professeurs les leur rappellerait, mais avec affection, et afin qu'un triste passé soit réparé par un bon avenir. La fête répond au besoin qu'a la jeunesse de délassement et de joie. [...] Le plaisir qu'on goûte, c'est celui que Dieu donne et qu'il permet, pour qu'un temps de relâche fasse diversion à la fatigue de l'esprit ${ }^{59}$.

Ces excursions peuvent aussi revêtir un caractère pacificateur, comme le suggère le récit d'une sortie en Drôme, où le goûtercerises fut 1'occasion de réconcilier les élèves de Poët-Laval, en rivalité avec ceux de Dieulefit ${ }^{60}$. Sans connaître les résultats effectifs à long terme (aucune cause objective de conflit n'est explicitée), la volonté d'apaiser les tensions entre les groupes d'enfants de deux villages est à relever. Ce récit présuppose qu'en matière de relations humaines, le choix d'un cadre joyeux et détendu prédispose mieux le cœur au pardon et à l'accueil de l'autre qu'une leçon de morale ou une confrontation dans le bureau de directeur de l'École. Gauthey

\footnotetext{
${ }^{56}$ Gauthey, 1861, p. 12.

${ }^{57}$ Gauthey, 1858, p. 185.

${ }^{58}$ Gauthey, 1858, p. 169.

${ }^{59}$ Gauthey, 1860, p. 43.

${ }^{60}$ Anonyme, 1856.
} 
affirmait ainsi : « la joie dilate le cœur, l'ouvre aux émotions nobles et sympathiques, tend à développer les sentiments bienveillants ${ }^{61} \gg$.

Une exhortation portant plutôt sur un thème de circonstance était apportée le plus souvent par le directeur de l'ÉdD. De même que le sapin jouait le rôle de symbole de la première fête de l'ÉdD, les élèves ont ici recours a un signe-symbole, d'identification et de ralliement : une bannière.

Ces sorties au vert sont aussi le reflet d'une époque où les idées hygiénistes du XIX ${ }^{\mathrm{e}}$ siècle annoncent la philosophie naturiste qui se développera plutôt au $\mathrm{XX}^{\mathrm{e}}$ siècle $^{62}$.

Le primat de récréation active va aussi profiter du développement des «nouvelles technologies » d'alors, comme ce fut le cas en Angleterre pour Thomas Cook (1808-1892). Ce baptiste, ébénisteévangéliste, devint voyagiste. Il fonda à Leicester en 1841 sa première agence de voyage ${ }^{63}$, tirant profit du développement des lignes ferroviaires. Cook sut négocier auprès de John Fox Bell, responsable de la Midland Counties Railway Company, un tarif très attractif d'un shilling par personne pour un aller-retour LeicesterLoughborough. Ainsi, il loua un train spécial pour offrir à d'anciens alcooliques et sympathisants (570 personnes en tout!) une journée d'excursion organisée par une Société de tempérance. Le premier voyage en train eut lieu le 5 juillet 1841, de Leicester à Loughborough, soit $19,5 \mathrm{~km}^{64}$. À sa façon, T. Cook cherchait à offrir une saine récréation aux ouvriers qu'il arrachait aux pubs où ils passaient leur dimanche à paresser, comme Raikes, en fondant les ÉdD en Angleterre, voulait arracher les enfants-ouvriers aux mauvaises compagnies de la rue, leur permettant à tous de tirer le meilleur parti du jour de repos hebdomadaire en leur offrant une activité adaptée.

\section{CONCLUSION}

Ces trois fêtes, rythmant régulièrement le calendrier annuel des acteurs des ÉdD, témoignent toutes d'une volonté de cultiver les liens relationnels intergénérationnels, terreau affectif propice à une active croissance de l'enfant avec les autres. Contrairement aux idées reçues, ce n'est pas l'austérité mais le primat de la joie qui prédomine chez ces protestants qui reconnaissent pourtant la doctrine du péché originel et de la «dépravation complète » de l'homme

\footnotetext{
${ }^{61}$ Gauthey, 1861, p. 13.

${ }^{62}$ Sur l'histoire de l'hygiénisme et du naturisme voir Bourdelais, 2001 ; Baubérot, 2004 ; Villaret, 2005.

${ }^{63}$ Simmons, 1973-1974.

${ }^{64}$ Cherrington, 1930 , p. $710-712$.
} 
selon Calvin, appellent à la «conversion » et à la foi au Christ rédempteur et fondent leur joie dans la grâce efficace, que la Bible seule leur révèle et qui nourrit leur foi en leur Sauveur. Point de kermesse, de tombola ou de vente de charité : c'est la gratuité du don, d'abord du don de soi par le service volontaire bénévole ${ }^{65}$ pour l'accueil de l'autre et pour lui permettre de grandir avec d'autres, qui prime.

Au cœur du dispositif, ce ne sont ni les savoirs ni les savants qui trônent, mais Dieu qui distribue les talents selon son entendement. D'où la gêne, comme chez les Jansénistes de Port-Royal ${ }^{66}$, quant à l'objet et à la fonction de la récompense. Celle-ci encourage la qualité des efforts déployés, assimilés au travail, et non le plus haut degré de compétence acquise, ce dernier étant fondamentalement dépendant d'une donnée non maîtrisable. Chez Gauthey, comme chez Comenius (1592-1670), c'est la métaphore agricole qui rend compte de l'anthropologie : le créateur donne à chaque homme un capital de germes non identiques. Il revient aux éducateurs, en bons jardiniers, de placer l'enfant dans les meilleures conditions pour qu'en les enseignant ce potentiel puisse porter du fruit tout au long de la vie. En cela, l'éducation de tous et toutes est une nécessité fondée sur la doctrine de la Création. Elle répond à la vocation de l'homme plutôt qu'à un " dictat » institutionnel fondé sur les besoins de la société ou sur le rang social de la famille.

Les fêtes n'instituent pas de rites qui auraient leur propre efficacité vitale. Tout au plus pourrait-on évoquer la notion d'ÉdD comme « rite de passage », propédeutique à l'entrée au catéchisme ${ }^{67}$ dans le vœu émis au synode du Havre par le pasteur Wilfred Monod. En cherchant à valoriser les connaissances, il faisait de l'ÉdD un pré-requis nécessaire à l'entrée au catéchisme. En proposant d'imposer l'ÉdD comme une instruction religieuse obligatoire au moins pour les enfants les plus pauvres dont les parents attendaient

\footnotetext{
${ }^{65}$ Avec l'expansion du Mouvement, de 1786 à 1800, l'Union anglaise avait vu son budget exploser. Ainsi, « Mise en difficulté financière, l'œuvre déclina faute de pouvoir payer les maîtres. À Gloucester même, trente ans après le début de l'œuvre, les écoles fermèrent en nombre important. Il devenait impérieux de changer d'organisation et d'employer des enseignants bénévoles » (Gregory, 1880, p. 97-98). Six jeunes amorcèrent non sans résistance ce mouvement d'engagement bénévole à Gloucester (Massé, 1880, p. 24). En France, seul "l'agent missionnaire » sera rétribué. Le principe du bénévolat était la règle, Chabrand puis le premier CÉdD l'avait déjà inscrit dans leurs recommandations pratiques. Chabrand, 1817, p. 13 ; CÉdD, 1827, p. 13.

${ }_{66}$ Augustiniens, les Jansénistes disaient comme le rapporte Compayré, [1895], p. 132 : « Si Dieu a mis quelque bien dans l'âme d'un enfant, il faut l'en louer et garder le silence » et cela pour préserver l'élève de l'orgueil et de la «nonchalance ».

${ }^{67}$ Notons que le synode officieux de Haute-Ardèche suggérait lui aussi un examen des connaissances pour les jeunes de 10 à 13 ans fréquentant l'École du Dimanche, là où d'autres organisaient plutôt des concours : Vabres, 1902, p. 115 ; Monod, 1902, p. 418.
} 
l'aide matérielle de l'Église ${ }^{68}$, Monod faisait le l'ÉdD une monnaie d'échange et de pression sur certaines familles.

Dans le détail, et comme l'illustre le tableau qui suit, chaque fête revêtait un accent particulier au sein de ce que l'on peut considérer comme un cycle non exempt de complémentarité et de cohérence.

\begin{tabular}{|l|c|c|c|}
\hline & $\begin{array}{c}\text { Fête Générale } \\
\text { ou Fête du Cirque }\end{array}$ & Fête de l'Arbre & Fête Champêtre \\
\hline Époque & Printemps & Décembre - janvier & Été \\
\hline Lieu & Salle de spectacle & Église & Campagne \\
\hline Concerne & $\begin{array}{c}\text { Les ÉdD } \\
\text { et membres des CA }\end{array}$ & $\begin{array}{c}\text { Les élèves } \\
\text { et les spectateurs }\end{array}$ & $\begin{array}{c}\text { L'ensemble } \\
\text { des acteurs d'une ÉdD }\end{array}$ \\
\hline Symbole & $\begin{array}{c}\text { Point d'orgue de la } \\
\text { semaine des AG }\end{array}$ & Arbre & Nature \\
\hline Sens & $\begin{array}{c}\text { Anniversaire des } \\
\text { œuvres du Réveil }\end{array}$ & $\begin{array}{c}\text { Enseignement et } \\
\text { récompenses }\end{array}$ & $\begin{array}{c}\text { Récréation après le } \\
\text { travail }\end{array}$ \\
\hline Typologie & $\begin{array}{c}\text { Commémorer } \\
\text { «mémoire/histoire » }\end{array}$ & $\begin{array}{c}\text { Récompenser } \\
\text { « récompense/grâce » }\end{array}$ & $\begin{array}{c}\text { «écréer } \\
\text { "récréation/shabbat » }\end{array}$ \\
\hline Analogie & $\begin{array}{c}\text { Souccot } \\
\text { ou fête des tentes }\end{array}$ & $\begin{array}{c}\text { Hannouka } \\
\text { ou fête des lumières }\end{array}$ & Shabbat \\
\hline
\end{tabular}

À la trilogie : «commémorer, récompenser et récréer », que nous proposons pour mettre en évidence l'accentuation respective de chacune d'entre elles, nous appliquerons la typologie de la " mémoire/histoire », de la « récompense/grâce », et de la " récréation/shabbat» comme cadre participant à la structuration des apprentissages tout au long de l'année. Pour prolonger, on peut encore développer l'analogie de ces trois fêtes avec trois fêtes joyeuses structurant la vie des familles israélites : la fête de Souccot ou " fête des Cabanes », celle de la Dédicace ou « fête de la lumière » et le Shabbat. Nous n'en proposons qu'une rapide évocation pour suggérer un des héritages de la pédagogie chrétienne, où l'histoire et les fêtes structurent et fécondent le présent de la vie individuelle par le moyen des familles pour ne pas oublier la grandeur de Dieu $(P S$ 78,7) et faire mieux que les pères (Ps 78,8, 1 Co 10,6).

La fête Générale ou fête du Cirque valorise la mémoire, au sens du souvenir reconnaissant. Celle-ci renvoie aux réseaux historiques auxquels appartiennent les acteurs des ÉdD, ceux de la grande famille des autres œuvres, fruit du Réveil. Ce n'est pas le bâtiment

${ }^{68}$ «Ne pourront plus recevoir l'assistance : les familles protestantes qui n'enverraient pas leurs enfants aux Écoles du Dimanche ou Jeudi, ou à l'Instruction religieuse des catéchumènes » (Monod, 1903, titre V, «Les secours », art. 12, p. 7). 
« en dur » ou « de toile » (le cirque d'alors n'était pas sous tente !) qui fait l'analogie avec Souccot (Ex 23;Dt 16; Lv 23), mais plutôt l'importance accordée au pèlerin qui fête, avec les différentes générations de sa famille réunie, l'assistance divine tout au long de ses pérégrinations et la fin d'un cycle. Cette histoire n'a pas eu que des moments joyeux et faciles, mais Dieu a fait route avec les siens, les conduisant à destination; les rapports d'AG pouvaient aussi en témoigner. Point d'orgue de cette semaine de printemps, la joie émanant de la fête Générale était comparable à celle des ouvriers fêtant au milieu des prémices la " récolte».

La fête de l'arbre fait émerger la notion de récompense-grâce. Si elle fait écho à l'action éducative de la grâce dans l'acquisition des compétences, montrant que tout ne dépend ni de l'efficacité des éducateurs ni du bon travail de l'enfant, elle montre aussi l'effet vivifiant de l'approbation-bénédiction. Cette fête pourrait être lue en écho à celle de la Dédicace du Second Temple, qui sert de cadre à $J n$ 10. Commémorant la victoire militaire de Judas Maccabée sur Antiochus Épiphane ${ }^{69}$, cette fête rappelait surtout le « miracle de la fiole d'huile » que seule la tradition talmudique rapporte ${ }^{70}$. La scénarisation de l'allumage des lampes à Hannouka, symbolisant le miracle, signe pour les nations de la victoire de Dieu qui fait briller sa lumière dans les ténèbres ${ }^{71}$, n'est pas sans trouver d'échos dans la « leçon de chose » illustrée par l'illumination de l'arbre de Noël et dans la véritable lumière à laquelle elle renvoie selon Gauthey ${ }^{72}$. Partant du constat selon lequel le langage imagé est « la base du langage des peuples primitifs ${ }^{73} »$, Gauthey considère qu'il est de facto plus assimilable par les enfants, et que, parce qu'il excite différents sens, il laisse une impression plus « durable » sur les cœurs. Il ajoute qu'il " s'agit de réaliser l'idée d'une fête de famille » ${ }^{74}$.

La fête champêtre développe, quant à elle, le concept de récréation après le travail. Elle fait écho au repos comme cessation $\mathrm{du}$ travail régulier, pour laisser place à des réjouissances communautaires. Cette activité récréative hors école faisait partie intégrante du dispositif éducatif. Cette fête revêt certains aspects communs avec le shabbat. Ce signe de l'Alliance mosaïque (Ex 31,13-17; Ez 20,12) instituait une rupture avec le travail régulier qu'il couronnait. Ce

\footnotetext{
${ }^{69} \mathrm{Ce}$ récit ne figure pas dans les écrits canoniques ( $\left.1 M 4,36-59 ; 2 M 10,1-18\right)$.

${ }^{70}$ Selon cette tradition, la réserve d'huile retrouvée dans le Second Temple purifié aurait dû illuminer la lampe pendant un jour. Celle-ci brilla pendant une semaine. Talmud Bavli, Shabbat 21 b.

${ }^{71}$ L'hypothèse de l'influence chrétienne sur la tradition plus récente d'échanges de cadeaux à cette fête est parfois suggérée. Avril - Maisonneuve, 1993, p. 111.

${ }^{72}$ Gauthey, 1858, p. 179-183.

${ }^{73}$ Op. cit., p. 181.

${ }^{74}$ Op. cit., p. 180.
} 
jour de joie commémorait l'œuvre créatrice et libératrice de Dieu (Ex 20,11; Dt 5,15) et préfigure, d'un point de vue chrétien, le plein repos ouvert par le Christ (Col 2,16-17, He 4,8-10). La présence de la famille y est aussi souhaitée. Dans ses recommandations pratiques, Gauthey précise :

vous chercherez à les installer [les enfants] dans un local champêtre qui ne soit pas très éloigné de la ville ou du village, afin que les parents et les amis puissent venir les rejoindre ${ }^{75}$.

Au regard de la valeur ainsi accordée aux fêtes, on peut s'interroger sur la place que leur ménagent les dispositifs éducatifs actuels, alors que, dans les sections scientifiques les plus convoitées, l'utilité d'enseigner l'histoire fait débat tout comme l'appel à « faire mémoire » de la dernière lettre de Guy Môquet pour inspirer «l'amour» de certaines valeurs aux lycéens plutôt que d'en faire des historiens. Il convient de noter toutefois que, au XIX ${ }^{\mathrm{e}}$ siècle déjà, les protestants n'étaient pas unanimes sur la question.

Ainsi, la teneur des discours de François Guizot ${ }^{76}$, deuxième président de la Société d'Encouragement pour l'Instruction parmi les Protestants de France (SEIPPF), allait-elle à l'encontre des idées que la loi Ferry allait imposer en 1881-1882. De fait, les protestants n'étaient pas tous disciples des idées et des valeurs promues par les ÉdD et que défendirent encore Ferdinand Buisson (1841-1932), Jules Steeg (1836-1898) et Felix Pécaut (1828-1898).

Enfin, comment le modèle pédagogique de la SÉD a-t-il évolué à partir de 1902 et jusqu'à aujourd'hui, après les vœux émis par Wilfred Monod au synode du Havre? L'idée d'école où règne «l'esprit de famille », perceptible dans ces fêtes, a-t-elle été cultivée ? À tout le moins, l'étude des fêtes au sein des ÉdD du XIX ${ }^{\mathrm{e}}$ siècle ne plaide-t-elle pas pour une école plus « incarnée », où les relations interpersonnelles seraient plus nourries? De façon plus profonde, ne serait-ce pas la notion de famille qui serait à revisiter, afin de nourrir l'expression d'une joie simple, fondée sur ce type de relations profondes, reçues et non choisies, et qui pourrait encore être un des moyens d'exciter la jeunesse à l'étude et de lui inspirer l'amour de l'école? À une époque où les familles sont plus morcelées, le développement des réseaux sociaux sur Internet, des recherches généalogiques, mais aussi l'essor de rencontres conviviales comme "la fête des voisins " ${ }^{77}$ ou d'associations telles que celles de

\footnotetext{
${ }^{75}$ Op. cit., p. 181.

${ }^{76}$ Guizot, 1852, p. 12 ; Guizot, 1864, p. 12-17 ; Guizot, 1872, cité par Robert, 1881, p. 13-29.

${ }^{77}$ La fête des voisins s'inscrit dans le tissu des relations sociales de solidarité de quartier. Fondée en 1999 par Atanase Périfan dans le XVII ${ }^{\mathrm{e}}$ arrondissement de Paris pour vaincre
} 
« grand-parents de cœur ${ }^{78} »$, ne sont-ils pas autant d'indices d'un besoin relationnel et identitaire intergénérationel ? Mais si «toute famille tire son nom du Créateur », selon l'enseignement des ÉdD (Ep 3.14), ne se heurte-t-on pas à une épineuse difficulté idéologique : sur quels fondements l'éducation, qui s'efforce de faire abstraction des questions métaphysiques dans son dispositif d'instruction, les reléguant à la sphère privée pour rester " neutre », pourrait-elle mieux prendre les valeurs des familles en compte et coopérer de façon plus interdépendante?

\section{ABRÉVIATIONS}

$\begin{array}{ll}\text { AG } & \text { Assemblée générale } \\ \text { CÉdD } & \text { Comité d'Encouragement des Écoles du Dimanche } \\ \text { ÉdD } & \text { Écoles du Dimanche } \\ \text { JÉdD } & \text { Journal des Écoles du Dimanche (Paris, SÉD) } \\ \text { MagÉdD } & \text { Magasin des Écoles du Dimanche } \\ \text { MusE } & \text { Musée des Enfants } \\ \text { SÉD } & \text { Société des Écoles du Dimanche } \\ \text { SEIPPF } & \text { Société d'Encouragement pour l'Instruction parmi les Protestants de France } \\ \text { SHPF } & \text { Société de l'Histoire du Protestantisme Français } \\ \text { UCJG } & \text { Union Chrétienne de Jeunes Gens }\end{array}$

\section{BIBLIOGRAPHIE}

Anonyme, 1818 : «Sur le Chant Religieux des Églises Protestantes françaises », in : Archives du Christianisme au XIX ${ }^{e}$ siècle, t. 1, Paris, Meyrueis, 1818, p. 344-345.

Anonyme, 1856: «Dieu-le-Fit (Drôme), "Promenade accompagnée d'un goûter de cerises" ", MagÉdD, 1856, p. 242.

Anonyme, 1858 : «Fête de Noël au Gymnase de M. Triat le 24 décembre 1857 », $M a g E ́ d D, 1858$, p. 47.

l'isolement des habitants et tisser des liens par le moyen de " pots » conviviaux la " fête des voisins " s'étend dès l'an 2000 sur toute la France avant de s'exporter à Bruxelles, Berlin, Genève, Dublin, Rome, Luxembourg, Lisbonne, Nicosie, Vienne, Londres, Brême au Canada, la Turquie, l'Ukraine, le Japon et Taïwan. En 2009, l'association estimait que 8,5 millions d'Européens dont 6,5 millions de Français avaient pris part à cette manifestation. Voir http://www.immeublesenfete.com.

${ }^{78}$ Le concept de « grands-parents de cœurs » ou « super-grandparents » est une réponse à l'éclatement des familles et aux besoins affectifs. Christelle Levasseur, qui a fondé cette association en 2007, fut à la fois marquée par l'apport structurant de sa grand-mère maternelle lorsque ses parents divorçaient et la peine de ne pouvoir offrir pareil cadre relationnel à ses enfants mais reconnaissante pour la compensation offerte par des proches. L'association met en relation des aînés souvent sans petits enfants avec des familles isolées. "Supergrandparents a pour vocation de rapprocher, d'une part des seniors en mal de petits-enfants et, d'autre part, des parents désireux de créer ou de recréer des liens pour leurs enfants qui sont malheureusement en rupture de relation grand parentale, quelles qu'en soient les raisons » (http://www.super-grandparents.fr/FR/whois_super_grandparents.awp). 
Anonyme, 1898 : Anonyme, « Notes de la rédaction », JÉdD, 1898, p. 413.

Avril - Maisonneuve, 1993 : A.-C. Avril - D. de La Maisonneuve (éd.), Les fêtes juives, Paris, Cerf, 1993 (Supplément - Cahiers Évangile, 86).

Baubérot, 2004 : A. Baubérot, Histoire du naturisme : le mythe du retour à la nature, Rennes, Presses universitaires de Rennes, 2004.

Blocher, 1979 : H. Blocher, Révélation des origines, Lausanne, Presses Bibliques Universitaires, 1979.

Boon, 1978 : B. Boon, Sing the Happy Song! A History of Salvation Army Vocal Music, London, Salvationist Publishing and Supplies, 1978.

Borel, 1864 : E. Borel, Statistique des associations Protestantes religieuses et charitables de France suivie d'un tableau des lieux de culte, écoles, \&c. de toutes les Églises protestantes de Paris, Paris, Ch. Maréchal, 1864.

Bost, 1854 : A. Bost, Mémoires pouvant servir à l'histoire du réveil religieux des Églises protestantes de la Suisse et de la France, t. 1, Paris, C. Meyrueis, 1854.

Bourdelais, 2001: P. Bourdelais, Les Hygiénistes, Enjeux, modèles et pratiques (XVIII ${ }^{e}-X X^{e}$ siècles), Paris, Belin, 2001.

Cadoret, 1808 : L. Cadoret, « Mon très cher et honoré frère en NSJC », Mss Luneray le 6 juin 1808 (SHPF, Mss nº E 290-30).

CÉdD, 1827: Comité d'Encouragement des Écoles du Dimanche, Conseils pour l'établissement et l'organisation des Écoles du Dimanche, Paris, Smith, 1827.

Chabrand, 1817 : D.-C. Chabrand, Des écoles du dimanche, de leur importance et de la manière de les diriger, Toulouse, A. Navarre, 1817.

Chalmel, 2004 : L. Chalmel, Réseaux philanthropinistes et pédagogie au $18^{e}$ siècle, Bern, Peter Lang, 2004.

Cherrington, 1930 : art. « Cook », in : E. H. Cherrington (éd.), Standard Encyclopedia of the Alcohol Problem, vol. 2, Westerville (OH), 1930, p. 710-712.

Collectif, 1898 : Collectif, « Notre enquête sur les arbres de Noël», JÉdD, 1898, p. 465-469.

Compayré, [1895]: G. Compayré, Histoire de la pédagogie, 29éd., Paris, Paul Mélottée, s. d. [1895] ( $1^{\text {re }}$ éd., vers 1880).

Cook, 1847 : J.-P. Cook, Histoire et organisation d'une École du Dimanche avec quelques conseils à ceux qui donnent aux enfants une instruction religieuse, Paris Nîmes - Lausanne, Delay - Garve - Bridel, 1847.

Cook, 1857 : J.-P. Cook, «Une fête des Écoles du Dimanche de Paris », MagÉdD, 1857, p. 103-114.

Cook, 1862 : J.-P. Cook, La vie de Charles Cook, t. 1, Paris, Librairie évangélique, 1862 .

Cramer, 1859: M. Cramer, «Nos Écoles du Dimanches: Les arbres de Noël», MagÉdD, 1859, p. 74-76.

Cullmann, 1993 : O. Cullmann, La Nativité et l'arbre de Noël. Les origines historiques, trad. de l'allemand, Paris, Cerf, 1993.

Fath, 2008 : S. Fath, Dieu XXL. La révolution des megachurches, Paris, Autrement, 2008.

Gauthey, 1858 : L. F. F. Gauthey, Essai sur les écoles du dimanche, Paris, Agence de la Société des écoles du dimanche, 1858. 
Gauthey, 1860 : L. F. F. Gauthey, De la vie dans les études ou Essai sur les moyens d'exciter la jeunesse au travail et de lui inspirer l'amour de ses devoirs, Paris, Meyrueis, 1860.

Gauthey, 1861 : L. F. F. Gauthey, Le délassement après le travail, ou Essai sur les récréations de l'enfance et de la jeunesse, Paris, Meyrueis, 1861.

Gonin, 1963 : G. Gonin, "Tradition protestante et transplantation », Archives des Sciences Sociales des Religions n ${ }^{\circ}$ 15, janvier-juin 1963, p. 122-136.

Gregory, 1880 : L. A. Gregory, Robert Raikes : journalist and philanthropist. A history of the origin of Sunday-Schools, London, Hodder, 1880.

Guizot, 1852 : F. Guizot, $A G$ SEIPPF, $1^{\text {er }}$ mai 1852.

Guizot, 1864 : F. Guizot, $A G$ SEIPPF, 9 avril 1864.

Guizot, 1872 : F. Guizot, $A G$ SEIPPF, 20 avril 1872.

Kuen, 2009 : A. Kuen, Musiques. I. Évolution de David à nos jours, Saint-Légier, Émmaüs, 2009.

Lelièvre, 1899 : M. Lelièvre, « Les Arbres de Noël », JÉdD, 1899, p. 489-490.

Léonard, 1953 : É.-G. Léonard, Le Protestant français, Paris, PUF, 1953.

M., 1861 : M.-A. M., « Ce que dit aux instructeurs l'arbre de Noël », MagÉdD, 1861, p. 46-48.

Massé, 1880 : A. Massé, Il y a cent ans. Origine des Écoles du dimanche, Lausanne Paris, Mognot - Bonhoure, 1880 (SHPF, T 552/969).

Mecklembourg-Schwerin, 1859 : Lettres originales de Madame la Duchesse d'Orléans, Hélène de Mecklenbourg-Schwerin [sic], et souvenirs biographiques, recueillis par G. H. de Schubert, Genève, Georg, 1859.

Minault, 1862 : P. Minault, « Société des Écoles du Dimanche », MagÉdD, 1862, p. 234.

Monod, 1902 : W. Monod, «L'instruction religieuse à l'École du Dimanche. Rapport lu au synode particulier du Havre en mai 1902 », Les Cahiers du Christianisme Social, $15^{\mathrm{e}}$ année, 1902, p. 418.

Monod, 1903 : W. Monod, «Église réformée de Rouen. Règlement», in : Diaconat, exercice 1903, Rouen, Blondel, 1903.

Monod, 1904 : W. Monod, Les Écoles du Dimanche en Normandie, Vals-les-Bains, Aberlen, 1904.

Montandon, 1864 : L. Montandon, «Avant-propos de la $1^{\text {re }}$ Édition» [1860], in : Chants religieux à l'usage des Écoles du Dimanche et des familles, $2^{\mathrm{e}}$ éd., Paris Strasbourg, Berger-Levrault, 1864, p. IV-VI.

Paumier, 1893 : H. Paumier, "Société des Écoles du Dimanche de France », in: F. Puaux (dir.), Exposition Universelle de Chicago. Les ouvres du protestantisme Français au XIX siècle, Paris, Comité Protestant Français, 1893, p. 350-356.

Père de famille, 1863 : Un père de famille, Les cirques, Paris, SÉD, 1863.

Pujade-Renaud, 2006 : C. Pujade-Renaud, L'école dans la littérature, Paris, L'Harmattan, 2006.

Ripert, 1956 : P. Ripert, Les origines de la crèche provençale et des santons populaires à Marseille, $2^{\mathrm{e}}$ éd., Marseille, Tacussel, 1956.

Ripoull, 2007 : J. Ripoull, Les bons comptes de Noël ou la fête qui ne fait pas de cadeau, Paris, Rocher, 2007. 
Robert, 1881 : Ch. Robert, $A G$ SEIPPF, 7 mai 1881, p. 13-29.

Rouillard, 2003 : P. Rouillard, Les fêtes chrétiennes en occident, Paris, Cerf, 2003.

Sautter, 1891 : L. Sautter, AG SÉD, 1891, p. 6-7.

Sautter, 1899 : L. Sautter, « Les Arbres de Noël », JÉdD, 1899, p. 49-53.

Sautter, 1900 : L. Sautter, $A G S E ́ D, 1900$, p. 10.

SÉD, 1897 : Prospectus, fête du Trocadéro, 24 juin 1897 (SHPF, SEIPPF 0Y7).

Simmons, 1973-1974 : J. Simmons, "Thomas Cook of Leicester», Transactions of the Leicestershire Archeological and Historical Society ${ }^{\circ} 49,1973-1974$, p. 18-32.

Troger, 2008 : V. Troger, "Pourquoi noter les élèves », Sciences Humaines $n^{\circ}$ 192, avril 2008, p. 41-43.

Trumbull, 1888 : H. C. Trumbull, The Sunday-School. Its Origin, Mission, Methods, and Auxiliaries, Philadelphia, Wattles, 1888.

Vabres, 1902 : A. D. Vabres, « Rapport sur les vœux et résolutions des synodes particuliers ", in: Actes et décisions du synode général officieux des Églises Réformés [Évangéliques] de France tenu à Anduze du 24 Juin au 2 juillet 1902, Alençon, Commission permanente du Synode, 1902.

Valdez, 1899 : A. Valdez, «Lettre d'Argenteuil, le 8 novembre 1899 sur les fêtes de l'arbre de Noël », JÉdD, 1899, p. 490-491.

Villaret, 2005 : S. Villaret, Histoire du naturisme en France depuis le siècle des Lumières, Paris, Vuibert, 2005.

Voigt, 2007 : K. H. Voigt, Internationale Sonntagsschule und deutscher Kindergottesdienst, Göttingen, Vandenhoeck \& Ruprecht, 2007.

Wargenau-Saillens, 1947 : M. Wargenau Saillens, Ruben \& Jeanne Saillens, évangélistes, Paris, Les Bons Semeurs, 1947.

Zorn 1996 : J.-F. Zorn, «Un mouvement catéchétique contemporain : les Écoles du Dimanche », ETR, 71, 1996/3, p. 379-400.

\section{Webographie}

Ville-Sélestat, [2009] : Office de la culture de la ville de Sélestat, Noël à Sélestat, 27 novembre 2009 - 6 janvier 2010, dossier de Presse $=\mathrm{http}: / /$ www.ville-selestat.fr/ spip_habitant/IMG/pdf/dp_noel2009.pdf [site consulté le 26 décembre 2009]. 